$09,12,04$

\title{
Механизмы термостимулированной люминесценции в УФ-облученных нанотрубках диоксида циркония
}

\author{
(C) И.А. Петренёв ${ }^{1}$, А.С. Вохминцев ${ }^{1}$, С.А. Старовойтова ${ }^{1}$, И.А. Вайнштейн ${ }^{1,2}$ \\ ${ }^{1}$ Уральский федеральный университет, \\ Екатеринбург, Россия \\ ${ }^{2}$ Институт металлургии УрО РАН, \\ Екатеринбург, Россия \\ E-mail: i.a.petrenev@urfu.ru
}

Поступила в Редакцию 8 июля 2021 г.

В окончательной редакции 13 июля 2021 г.

Принята к публикации 16 июля 2021 г.

Нанотубулярный слой диоксида циркония синтезирован методом анодного окисления. Изучены кривые спектрально-разрешенной термостимулированной люминесценции в области 390-550 nm после возбуждения УФ-излучением с длиной волны $300 \mathrm{~nm}$. Выполнена оценка энергетических и кинетических параметров кривых термостимулированной люминесценции. Предложен механизм термоактивационных процессов с участием собственных дефектов решетки.

Ключевые слова: спектрально-разрешенная термостимулированная люминесценция, диоксид циркония, нанотрубки, электронные и дырочные ловушки.

DOI: $10.21883 /$ FTT.2021.11.51580.09s

\section{1. Введение}

Нанопористрые и нанотубулярные массивы диоксида циркония $\left(\mathrm{ZrO}_{2}\right.$-nt) используются в различных отраслях науки и техники благодаря высокой химической, механической, температурной стабильности и площади поверхности [1-3]. На их основе производятся батареи, конденсаторы, топливные элементы, твердые электролиты, катализаторы, дозиметры и мемристоры [4-7].

Известно, что точечные дефекты (F-, $\mathrm{F}^{+}$- и $\mathrm{Zr}^{3+}$-центры) и их агрегаты (например, Т-дефекты [8], вакансионные кластеры [9] и т.д.) являются оптически активными центрами и формируют особенности отклика диоксида циркония на различные внешние воздействия. Ранее нанотрубки $\mathrm{ZrO}_{2}$-nt, полученные методом анодирования, были изучены с использованием спектральных методик фотолюминесценции (ФЛ) [10-17] и гальванолюминесценции [18]. Кроме того, в ряде работ методами термостимулированной люминесценции (ТСЛ) в спектральной области 400-650 nm были исследованы порошки моноклинного $\mathrm{ZrO}_{2}$ в исходном состоянии и после высокотемпературных обработок на воздухе [7,9-24], в вакууме [35] или в атмосфере аргона [26]. Были получены экспериментальные подтверждения наличия ловушек носителей заряда, активных ниже комнатной температуры (223-293 K) [26] и в широком температурном диапазоне от 300 до $750 \mathrm{~K}$ [7,19-25] после облучения образцов электронным пучком $[7,19,25], \quad \beta-[20-23]$ или УФ-излучением $[24,26]$. Исследования нанотубулярных структур диоксида циркония методом ТСЛ к настоящему времени не известны.

Таким образом, цель данной работы заключалась в изучении термоактивационных процессов в синтезиро- ванных массивах нанотрубок $\mathrm{ZrO}_{2}$ после воздействия УФ-излучением.

\section{2. Образцы и методы}

Нанотубулярный массив диоксида циркония $\mathrm{ZrO}_{2}$-nt был синтезирован методом анодирования Zr-фольги с примесью $\mathrm{Hf}<1 \%$ [27]. Zr-фольга толщиной $100 \mu \mathrm{m}$ предварительно обезжиривалась, погружалась в ультразвуковую ванну, обрабатывалась раствором кислот $\mathrm{HF}: \mathrm{HNO}_{3}: \mathrm{H}_{2} \mathrm{O}=1: 6: 20$, промывалась дистиллированной водой и сушилась на воздухе. Анодирование проводилось в двухэлектродной электрохимической ячейке при постоянном напряжении $20 \mathrm{~V}$ и температуре анода $10^{\circ} \mathrm{C}$ в течение $6 \mathrm{hr}$.

Электролит представлял собой раствор этиленгликоля, содержащий 5 wt.\% $\mathrm{H}_{2} \mathrm{O}$ и 1 wt.\% $\mathrm{NH}_{4} \mathrm{~F}$.

Морфологические параметры синтезированных слоев изучались на сканирующем электронном микроскопе (СЭM) SIGMA VP компании Carl Zeiss. Из СЭМ-снимка, представленного на рис. 1, видно, что слой оксида состоит из самоупорядоченных нанотрубок диаметром $50 \mathrm{~nm}$. При анодном окислении $\mathrm{Zr}$-фольги в указанных выше условиях толщина синтезированных массивов $\mathrm{ZrO}_{2}$-nt составляет $\sim 5 \mu \mathrm{m}$ [27].

После синтеза слои $\mathrm{ZrO}_{2}$-nt нагревались до $800 \mathrm{~K}$ на воздухе для удаления остатков электролита. Образцы для ТСЛ-исследований представляли собой $\mathrm{Zr}$-подложку прямоугольной формы размерами $12 \times 7 \mathrm{~mm}$ со слоем $\mathrm{ZrO}_{2}$-nt с одной стороны металла.

Измерения кривых ТСЛ проводилось на люминесцентном спектрометре Perkin Elmer LS 55 с высо- 


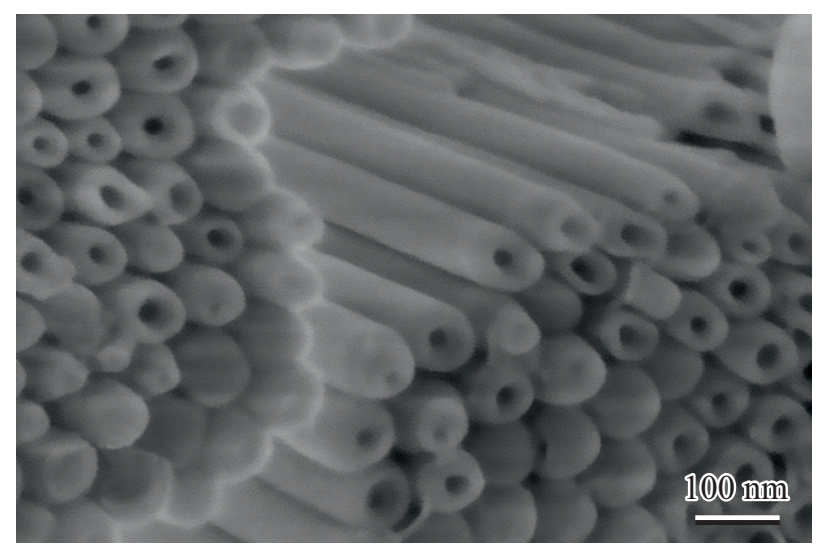

Рис. 1. Изображение синтезированных нанотрубок $\mathrm{ZrO}_{2}$-nt.

котемпературной приставкой [28]. Исследования ТСЛ выполнялись в диапазоне температур $T=300-773 \mathrm{~K}$ при скорости нагрева $\beta=2 \mathrm{~K} / \mathrm{s}$. Перед измерением ТСЛ образцы нагревались до $773 \mathrm{~K}$ для опустошения ловушек и остывали до комнатной температуры внутри закрытой от света камере спектрометра. После чего исследуемые образцы подвергались воздействию монохроматического УФ-излучения с длиной волны $\lambda=300 \mathrm{~nm}(4.1 \mathrm{eV})$ в течение $10 \mathrm{~min}$. при комнатной температуре. Доза УФ-излучения составляла $1 \mathrm{~mJ} / \mathrm{cm}^{2}$. Спектрально-разрешенная ТСЛ измерялась в диапазоне длин волн от 390 до $550 \mathrm{~nm} \mathrm{с} \mathrm{шагом}$ $20 \mathrm{~nm}$, щель в регистрирующего монохроматоре составляла $20 \mathrm{~nm}$.
Экспериментальные кривые ТСЛ анализировались в рамках формализма кинетики общего порядка [29]:

$$
\begin{aligned}
I(T)= & s^{\prime \prime} n_{0} \exp \left(-\frac{E_{\mathrm{a}}}{k T}\right) \\
& \times\left[1+\frac{s^{\prime \prime}(b-1)}{\beta} \int_{T_{0}}^{T} \exp \left(-\frac{E_{\mathrm{a}}}{k T^{\prime}}\right) d T^{\prime}\right]^{\frac{b}{b-1}},
\end{aligned}
$$

где $I(T)$ - интенсивность ТСЛ; $s^{\prime \prime}$ - эффективный частотный фактор, $\mathrm{s}^{-1} ; n_{0}-$ начальная концентрация заполненных ловушек после облучения, $\mathrm{m}^{-3} ; E_{\mathrm{a}}-$ энергия термической ионизации, $\mathrm{eV} ; k-$ постоянная Больцмана, eV/K; $T$ - температура, $\mathrm{K} ; b-$ порядок кинетики; $\beta-$ скорость нагрева, $\mathrm{K} / \mathrm{s} ; T_{0}=300 \mathrm{~K}-$ начальная температура, K.

Энергетические и кинетические характеристики центров захвата определялись методом аппроксимации формы ТСЛ-кривых суперпозицией нескольких независимых пиков общего порядка кинетики согласно выр. (1). Данная методика успешно применяется в ТСЛ дозиметрии ионизирующих излучений при исследовании широкозонных материалов [20-23,30,31].

\section{3. Результаты и обсуждение}

\section{1. Кривые ТСЛ в различных полосах свечения}

На рис. 2 изображены кривые ТСЛ, измеренные в исследуемых полосах свечения. Видно, что все термо-

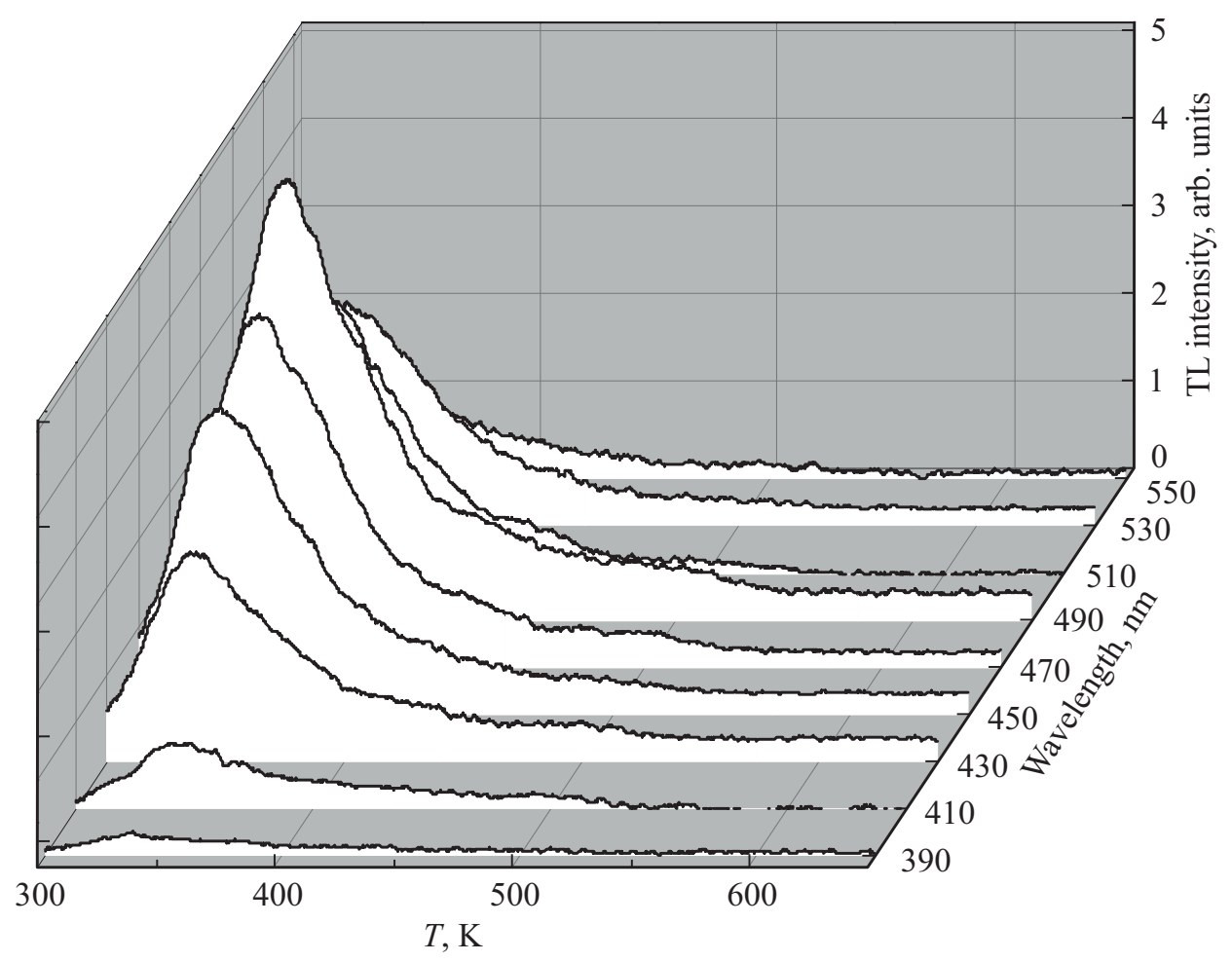

Рис. 2. Экспериментальные кривые ТСЛ при различных длинах волн регистрации. 
активационные кривые имеют один пик с максимумом при температуре около $340 \mathrm{~K}$, а в области $400-550 \mathrm{~K}$ для каждой из них регистрируется менее интенсивное плечо. Отметим, что все экспериментальные кривые в исследуемой спектральной области имеют схожую форму и что наибольшая интенсивность ТСЛ наблюдается в полосе $490 \mathrm{~nm}$. Далее для интерпретации природы центров рекомбинации, участвующих в регистрируемых термоактивационных процессах в исследуемом температурном диапазоне, строился и анализировался спектр свечения ТСЛ.

\section{2. Спектральные характеристики тСл}

На рис. 3 изображена зависимость светосуммы ТСЛ от энергии фотонов для $\mathrm{ZrO}_{2}$-nt. Каждая точка спектра соответствует площади под экспериментальной ТСЛ-кривой (рис. 2) в указанном температурном диапазоне для исследуемых полос свечения.

Видно, что спектр ТСЛ удовлетворительно описывается двумя пиками гауссовой формы G1 и G2 с энергиями максимумов $E_{\max }=2.47 \pm 0.02$ и $2.72 \pm 0.03 \mathrm{eV}$ и полуширинами $\omega=0.23 \pm 0.04$ и $0.39 \pm 0.12 \mathrm{eV}$ соответственно. Полученные результаты согласуются с нашими исследованиями ФЛ-свойств нанотубулярных слоев $\mathrm{ZrO}_{2}[10]$, для которых регистрируются также две полосы свечения гауссовой формы с $E_{\max }=2.43 \pm 0.01$ и $2.68 \pm 0.03 \mathrm{eV}$, а также $\omega=0.35 \pm 0.01$ и $0.48 \pm 0.02 \mathrm{eV}$ соответственно. Кроме того, в независимых исследованиях образцов порошков моноклинного $\mathrm{ZrO}_{2}$ в спектрах ТСЛ [7,19-26], ФЛ [7,23,25,26] и импульсной катодолюминесценции $[7,25]$ регистрируется широкая неэлементарная полоса свечения в диапазоне 400-650 nm $(3.10-1.91 \mathrm{eV})$ с максимумом в области $480-500 \mathrm{~nm}$ $(2.58-2.48 \mathrm{eV})$.

Таким образом, близкий спектральный состав люминесценции при различных видах возбуждения $\mathrm{ZrO}_{2}$-nt и порошков моноклинного $\mathrm{ZrO}_{2}$ может указывать на преобладание моноклинной фазы в исследуемых образцах. Указанный факт также согласуется с исследованиями нанотрубок $\mathrm{ZrO}_{2}$-nt методом рентгеновской дифракции, для которых регистрируется образование моноклинной фазы в результате отжига при $400^{\circ} \mathrm{C}$ в течение $1 \mathrm{hr}$ на воздухе [32]. В то же время можно сделать вывод об участии двух центров излучательной рекомбинации в исследуемых термоактивационных процессах.

Анализ литературы показал, что свечение в полоce G1 $(\sim 2.4 \mathrm{eV})$ связано с рекомбинацией дырок из валентной зоны с электронами, захваченными ионами $\mathrm{Zr}^{3+}[8]$. О природе полосы $\mathrm{G} 2(2.5-2.7 \mathrm{eV})$ на сегодняшний день нет единого мнения [25]. Одни связывает люминесценцию с релаксацией примесных ионов $\mathrm{Ti}^{3+}$, которые могут присутствовать в $\mathrm{ZrO}_{2}$ в очень низкой концентрации [33]. В нашем случае наличие в исследуемых образцах примесных ионов Ті исключено на этапе синтеза. Другие - с релаксацией кислородных вакансий в объеме или на поверхности образца [34,35]. Имеются

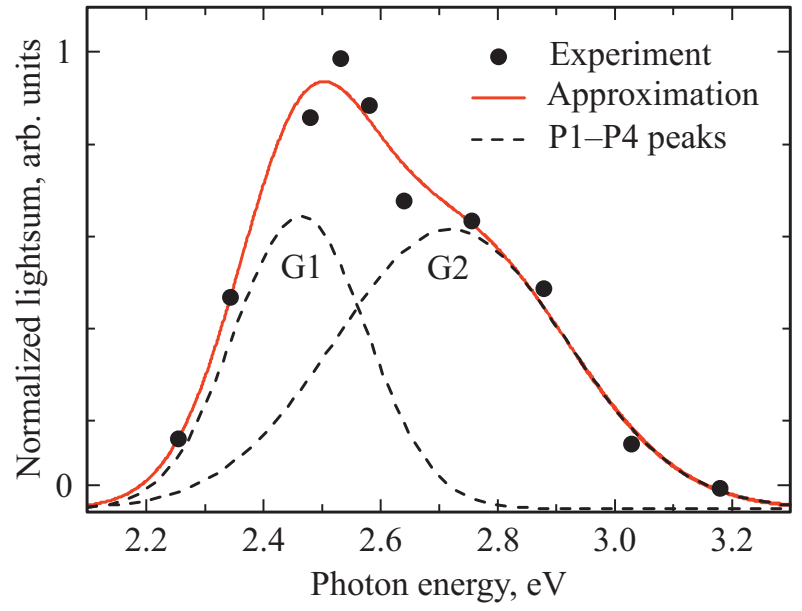

Рис. 3. Разложение спектра ТСЛ на пики гауссовой формы.

также мнения об участии комплексных дефектов, включающими в себя ассоциированные кислородные вакансии [36] или ионы $\mathrm{Zr}^{3+}$, связанные с двумя соседними вакансиями кислорода (Т-центрами) [8].

Возможное участие Т-центров в механизмах регистрируемой ТСЛ удовлетворительно согласуется с положением уровня возбужденного состояния Т-дефектов, который находится на $4.2 \mathrm{eV}$ выше потолка валентной зоны [8]. Отметим, что используемый метод синтеза образцов $\mathrm{ZrO}_{2}$-nt и их развитая поверхность способствует накоплению большого числа кислородных вакансий и ионов $\mathrm{Zr}^{3+}$ соответственно. В то же время повышается вероятность формирования Т-центров, что подтверждается сравнимыми интенсивностями гауссовых компонент в спектре ТСЛ (рис. 3).

\section{3. Оценки энергетических и кинетических параметров}

На рис. 4 показаны аппроксимации экспериментальных кривых ТСЛ в полосах $\lambda_{\text {em }}=490$ и $430 \mathrm{~nm}$, относящихся к свечениям в областях G1 и G2 соответственно. Разложения выполнены с использованием суперпозиции 4 пиков (P1-P4) общего порядка кинетики согласно выр. (1). В таблице приведены полученные значения параметров аппроксимации с коэффициентами детерминации $R^{2}>0.999$.

Анализ таблицы показал, что значения $T_{\max }$ для ТСЛ-пиков в полосе $\lambda_{\mathrm{em}}=430 \mathrm{~nm}$ сдвинуты в низкотемпературную область на $2-10 \mathrm{~K}$ относительно значений $T_{\max }$ соответствующих пиков в полосе $\lambda_{\mathrm{em}}=490 \mathrm{~nm}$. В механизмах ТСЛ, ответственных за пики Р1-P3, доминируют процессы второго порядка кинетики, а за пики P4 - первого. Все наблюдаемые пики характеризуются близкими значениями $E_{\mathrm{a}}$. Для пиков Р1 и Р2 значения эффективного частотного фактора изменяется в диапазоне $s^{\prime \prime}=(8.5-6.2) \cdot 10^{9} \mathrm{~s}^{-1}$, а для пиков Р3 и P4 $-s^{\prime \prime}=(9.7-0.9) \cdot 10^{7} \mathrm{~s}^{-1}$. Таким образом, одинаковый характер описания кривых ТСЛ говорит о том, 
что за изучаемые ТСЛ-процессы в исследуемых полосах свечения отвечают одни и те же ловушки носителей заряда.

Полученные значения параметров термоактивационных процессов (таблица) удовлетворительно согласуются с ТСЛ-исследованиями порошка моноклинного $\mathrm{ZrO}_{2}$ в полосе $350-450 \mathrm{~nm}$ после $\beta$-облучения дозой $10 \mathrm{~Gy}$ [22]. В данной работе кривые ТСЛ были описаны четырьмя пиками первого порядка кинетики с близкими значениями $E_{\mathrm{a}}=0.69-0.74 \mathrm{eV}$ и $s=3 \cdot 10^{8}-9 \cdot 10^{9} \mathrm{~s}^{-1}$. Авторы работы [22] связали регистрируемые ТСЛ-пики с четырьмя электронными ловушками.

Отметим, что в нашем случае значения параметра $s^{\prime \prime}$ для пиков Р1 и Р2 на два порядка выше аналогичных значений для пиков Р3 и Р4 при близких значениях $E_{\mathrm{a}}=0.71-0.80 \mathrm{eV}$ и изменении значений $T_{\max }$ пиков от 334 до $496 \mathrm{~K}$. По данным работ [37,38], кислородные вакансии в $\mathrm{ZrO}_{2}$ являются электронными ловушками с $E_{\mathrm{a}} \approx 0.7 \mathrm{eV}$. В то же время из расчетной работы [39] междоузельные ионы $\mathrm{O}_{i}^{2-}$, находящиеся в окружении трех $\mathrm{Zr}$-ионов, являются дырочными ловушками и имеют энергию сродства к дырке, равную $0.78 \mathrm{eV}$. Это, в свою очередь, также соответствует диапазону полученных значений $E_{\mathrm{a}}$ для пиков $\mathrm{P} 1-\mathrm{P} 4$ (таблица).
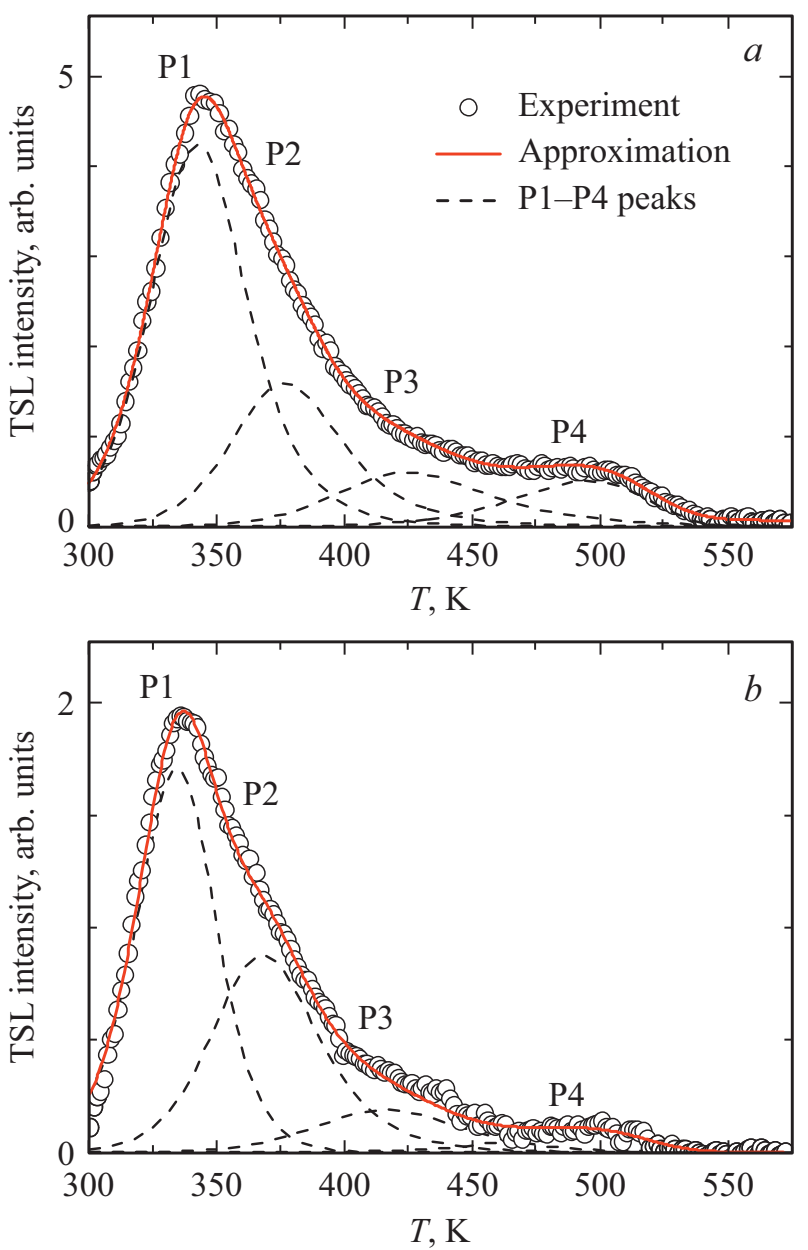

Рис. 4. Разложение кривой ТСЛ в полосах $a) 490$ и $b) 430 \mathrm{~nm}$ на элементарные компоненты общего порядка кинетики.
Значения температуры максимума $T_{\max }$, энергии ионизации $E_{\mathrm{a}}$, частотного фактора $s^{\prime \prime}$ и порядка кинетики $b$ для пиков P1-P4 в полосах 490 и $430 \mathrm{~nm}$

\begin{tabular}{c|c|c|c|c|c}
\hline Пик & $\lambda_{\mathrm{em}}, \mathrm{nm}$ & $T_{\max }, \pm 2 \mathrm{~K}$ & $E_{\mathrm{a}, \pm 0.03 \mathrm{eV}}$ & $s^{\prime \prime}, \mathrm{s}^{-1}$ & $b$ \\
\hline \multirow{2}{*}{ P1 } & 490 & 343 & 0.73 & $8.3 \cdot 10^{9}$ & 1.9 \\
\cline { 2 - 6 } & 430 & 334 & 0.76 & $8.5 \cdot 10^{9}$ & 1.6 \\
\hline \multirow{2}{*}{ P2 } & 490 & 377 & 0.80 & $8.0 \cdot 10^{9}$ & 2.0 \\
\cline { 2 - 6 } & 430 & 367 & 0.78 & $6.2 \cdot 10^{9}$ & 2.0 \\
\hline \multirow{2}{*}{ P3 } & 490 & 425 & 0.76 & $9.7 \cdot 10^{7}$ & 2.0 \\
\cline { 2 - 6 } & 430 & 416 & 0.71 & $2.1 \cdot 10^{7}$ & 1.8 \\
\hline \multirow{2}{*}{ P4 } & 490 & 496 & 0.80 & $8.6 \cdot 10^{6}$ & 1.1 \\
\cline { 2 - 6 } & 430 & 494 & 0.80 & $1.0 \cdot 10^{7}$ & 1.0
\end{tabular}

Таким образом, обнаруженные спектральные особенности ТСЛ и полученные параметры центров захвата (см. таблицу), а также их сравнение с независимыми экспериментальными и теоретическими работами [8,25,32-39] позволяют предложить модель механизмов для исследуемых ТСЛ-процессов в нанотубулярных массивах диоксида циркония с участием как электронных, так и дырочных ловушек.

При внутризонном возбуждении образцов $\mathrm{ZrO}_{2}$-nt монохроматическим УФ-излучением с энергией $4.1 \mathrm{eV}$ протекают следующие ТСЛ-процессы. При поглощении фотона с энергией $4.1 \mathrm{eV} e$ из валентной зоны переходит на возбужденный уровень Т-дефекта с последующей безызлучательной релаксацией $e$ на уровень $\mathrm{Zr}^{3+}$-центра. При этом образовавшаяся в валентной зоне $h$ захватывается междоузельным ионом $\mathrm{O}_{i}^{2-}$. Далее при повторном поглощении фотона с энергией $4.1 \mathrm{eV} e$ переходит в зону проводимости и захватывается кислородной вакансией $\left(\mathrm{V}_{\mathrm{O}}\right)$. Затем нагрев УФ-облученных образцов приводит к появлению свободных $e$ и $h$ в зоне проводимости и валентной зоне соответственно. В дальнейшем регистрируется излучательная рекомбинация с участием $\mathrm{Zr}^{3+}$-центров (в полосе $\mathrm{G} 1$ ), а также $\mathrm{F}-, \mathrm{F}^{+}$-центров и их комплексов (в полосе $\mathrm{G} 2$ ).

\section{4. Заключение}

Методом анодирования синтезированы нанотубулярные слои $\mathrm{ZrO}_{2}$-nt толщиной $5 \mu \mathrm{m}$ с внутренним диаметром нанотрубок $50 \mathrm{~nm}$. Измерена серия кривых ТСЛ в фиксированных полосах свечения в диапазоне 390-550 nm образцов $\mathrm{ZrO}_{2}$-nt после облучения УФ-излучением.

В спектре свечения ТСЛ обнаружены две компоненты гауссовой формы G1 и G2 с максимумами при 2.47 и $2.72 \mathrm{eV}$ соответственно. Анализ литературы показал, что свечение в полосе G1 связано с рекомбинацией дырок из валентной зоны с электронами, захваченными 
ионами $\mathrm{Zr}^{3+}$, а в полосе $\mathrm{G} 2$ - с участием $\mathrm{F}-, \mathrm{F}^{+}$-центров и их комплексов.

Выполнено разложение ТСЛ-кривых суперпозицией четырех пиков общего порядка кинетики. Определены энергетические и кинетические характеристики регистрируемых ТСЛ-процессов. Сделаны заключения, что электронными ловушками являются кислородные вакансии, а дырочными - междоузельные ионы кислорода со значениями энергий термической ионизации $E_{\mathrm{a}}=0.7-0.8 \mathrm{eV}$. На основе полученных данных и проведенного анализа литературы предложены механизмы наблюдаемых термоактивационных процессов в нанотрубках $\mathrm{ZrO}_{2}$ после воздействия УФ-излучением.

\section{Финансирование работы}

Работа выполнена в рамках научного проекта FEUZ-2020-0059 Министерства науки и высшего образования РФ.

\section{Конфликт интересов}

Авторы заявляют, что у них нет конфликта интересов.

\section{Список литературы}

[1] H. Tsuchiya, J.M. Macak, A. Glicov, L. Taveira, P. Schmuki. Corros. Sci. 47, 12, 3324 (2005).

[2] H. Tsuchiya, J.M. Macak, I. Sieber, P. Schmuki. Small 1, 7, 722 (2005).

[3] W.-J. Lee, W.H. Smyrl. Electrochem. Solid-State Lett. 8, 3, B7 (2005).

[4] D. Panda, T.-Y. Tseng. Thin Solid Films 531, 1 (2013).

[5] A.S. Vokhmintsev, R.V. Kamalov, A.V. Kozhevina, I.A. Petrenyov, N.A. Martemyanov, I.A. Weinstein. Proceed. USBEREIT 2018, 348 (2018).

[6] I.A. Petrenyov, A.S. Vokhmintsev, R.V. Kamalov, I.A. Weinstein. AIP Conf. Proceed. 2174, 020242 (2019).

[7] S.V. Nikiforov, V.S. Kortov, M.G. Kazantseva, K.A. Petrovykh. J. Lumin. 166, 111 (2015).

[8] V.M. Orera, R.I. Merino, Y. Chen, R. Cases, P.J. Alonso. Phys. Rev. B 42, 16, 9782 (1990).

[9] D.S. Aidhy, Y. Zhang, W.J. Weber. Scripta Mater. 98, 16 (2015).

[10] A.S. Vokhmintsev, R.V. Kamalov, I.A. Petrenyov, I.A. Weinstein. AIP Conf. Proceed. 2313, 030033 (2020).

[11] F. Trivinho-Strixino, F.E.G. Guimaräes, E.C. Pereira. Chem. Phys. Lett. 461, 1-3, 82 (2008).

[12] F. Trivinho-Strixino, F.E.G. Guimaräes, E.C. Pereira. Mol. Cryst. Liq. Cryst. 485, 766 (2008).

[13] X. Wang, J. Zhao, P. Du, L. Guo, X. Xu, C. Tang. Mater. Res. Bull. 47, 11, 3916 (2012).

[14] M. Wang, X. Wang, J. Lin, X. Ning, X. Yang, X. Zhang, J. Zhao. Ceram. Int. 41, 7, 8444 (2015).

[15] N. Fu, X. Wang, L. Guo, J. Zhao, X. Zhang, J. Lin, L. Cong, M. Wang, Y. Yang. J. Mater. Sci. Mater. Electron. 28, 10, 7253 (2017).

[16] D. Liu, M. Wang, L. Gong, J. Zhao, M. Zhu, X. Wang. J. Alloys Compd 864, 158781 (2021).
[17] E.-Y. Seo, S.-K. Choi, I.-S. Shin, W.-K. Kang. J. Kor. Chem. Soc. 57, 5, 547 (2013).

[18] S. Stojadinović, R. Vasilić, M. Petković, I. Belča, B. Kasalica, M. Perić, Lj. Zeković. Electrochim. Acta 79, 133 (2012).

[19] S.V. Nikiforov, V.S. Kortov, D.L. Savushkin, A.S. Vokhmintsev, I.A. Weinstein. Radiation Measurements 106, 155 (2017).

[20] H.S. Lokesha, K.R. Nagabhushana, F. Singh. J. Lumin. 192, 173 (2017).

[21] H.S. Lokesha, N. Chauman, K.R. Nagabhushana, F. Singh. Ceram. Int. 44, 15, 18871 (2018).

[22] H.S. Lokesha, M.L. Chithambo, S. Chikwembani. J. Lumin. 218, 116864 (2020).

[23] H.S. Lokesha, K.R. Nagabhushana, F. Singh. Opt. Mater. 107, 109984 (2020).

[24] Y. Cong, B. Li, B. Lei, W. Li. J. Lumin. 126, 2, 822 (2007).

[25] S.V. Nikiforov, A.A. Menshenina, S.F. Konev. J. Lumin. 212, 219 (2019).

[26] V. Kiisk, L. Puust, K. Utt, A. Maaroos, H. Mändar, E. Viviani, F. Piccinelli, R. Saar, U. Joost, I. Sildos. J. Lumin. 174, 49 (2016).

[27] I.A. Petrenyov, R.V. Kamalov, A.S. Vokhmintsev, N.A. Martemyanov, I.A. Weinstein. J. Phys. Conf. Ser. 1124, 2, 022004 (2018).

[28] A.S. Vokhmintsev, M.G. Minin, A.M.A. Henaish, I.A. Weinstein. Measurement 66, 90 (2015).

[29] S.W.S. McKeever, R. Chen. Radiation Measurements 27, 5-6, 625 (1997).

[30] I.A. Weinstein, A.S. Vokhmintsev, M.G. Minin, V.V. Kartashov, I.V. Chernetsky. Radiation Measurements 56, 236 (2013).

[31] A.S. Vokhmintsev, M.G. Minin, I.A. Weinstein. Radiation Measurements 106, 55 (2017).

[32] A.V. Kozhevina, A.S. Vokhmintsev, R.V. Kamalov, N.A. Martemyanov, A.V. Chukin, I.A. Weinstein. J. Phys. Conf. Series 917, 6, 062031 (2017).

[33] Y. Cong, B. Li, S. Yue, D. Fan, X. Wang. J. Phys. Chem. C 113, 31, 13974 (2009).

[34] T.V. Perevalov, D.V. Gulyaev, V.S. Aliev, K.S. Zhuravlev, V.A. Gritsenko, A.P. Yelisseyev. J. Appl. Phys. 116, 24, 244109 (2014).

[35] N.G. Petrik, D.P. Taylor, T.M. Orlando. J. Appl. Phys. 85, 9, 6770 (1999).

[36] N. Korsunska, V. Papusha, O. Kolomys, V. Strelchuk, A. Kuchuk, V. Kladko, Yu. Bacherikov, T. Konstantinova, L. Khomenkova. Phys. Status Solidi C 11, 9-10, 1417 (2014).

[37] C. Bettinali, G. Ferbaresso, J.W. Manconi. J. Chem. Phys. 50, 9, 3957 (1969).

[38] K.A. Shoaib, F.H. Hashmi, M. Ali, S.J.H. Bukhari, C.A. Majid. Phys. Status Solidi 40, 2, 605 (1977).

[39] A.S. Foster, V.B. Sulimov, F. Lopez Gejo, A.L. Shluger, R.M. Nieminen. Phys. Rev. B 64, 22, 224108 (2001).

Редактор Е.В. Толстякова 